\title{
UNIFORMITY OF RADIANT HEAT FLUXES IN CONE CALORIMETER
}

\author{
Michael T. Wilson, Bogdan Z. Dlugogorski and Eric M. Kennedy \\ Process Safety and Environment Protection Group \\ The University of Newcastle \\ Callaghan, NSW 2308, AUSTRALIA
}

\begin{abstract}
A model has been developed for the local configuration factor for the radiant interchange between the internal surface of the cone heater and the specimen's surface in the cone calorimeter. The incident heat flux under the cone was characterised in a series of experiments as a function of spatial coordinates. Both the emissivity and the temperature of the heating element were estimated from the experimental data. Significant variations in the surface temperature, and therefore in radiosity, were observed. The expression for the configuration factor was applied to estimate uncertainties in the measured variables due to tolerances in independent parameters. Plots were provided of the configuration factor and isolines of the radiant heat flux at various heights below the base of the cone. A comparison was carried out between predicted and measured data, demonstrating good agreement. The model predicted that, at the standard location of the specimen of $25 \mathrm{~mm}$ below the frustum, the radiant flux is uniform but only within the central area of the sample. Toward the edges and corners, the flux decreases by 2.6 and $15.9 \%$, respectively, in comparison to that along the centreline.
\end{abstract}

Keywords: cone calorimeter, view factor, radiant heat fluxes

\section{NOMENCLATURE}

$A_{3} \quad$ area of surface 3

$a$

distance from centreline

$d A_{1} \quad$ elemental area on the sample's surface

$F_{3-d 1} \quad$ configuration factor between surface 3 and elemental area $d A_{l}$

$F_{d 1-2} \quad$ configuration factor between elemental area $d A_{l}$ and surface 2

$F_{d 1-3} \quad$ configuration factor between elemental area $d A_{l}$ and surface 3

$F_{d 1-4} \quad$ configuration factor between elemental area $d A_{l}$ and surface 4

$H_{2}, H_{4} \quad$ parameters defined as $H_{2}=z / a$ and $H_{4}=(h+z) / a$, respectively

$h \quad$ height of the frustum ( $65 \mathrm{~mm}$ for standard cone)

$q$ " local radiant heat flux on the specimen's surface $\left(\mathrm{W} / \mathrm{m}^{2}\right)$

$R_{2}, R_{4} \quad$ parameters defined as $R_{2}=r_{2} / a$ and $R_{4}=r_{4} / a$, respectively

$r_{2}, r_{4} \quad$ radii of the base and top of the frustum $(80 \mathrm{~mm}$, and $40 \mathrm{~mm}$, respectively)

$T \quad$ average surface temperature of the heating element (K)

$z \quad$ vertical distance from the lower base of the frustum to the sample surface

$Z_{2}, Z_{4} \quad$ parameters defined as $Z_{2}=1+H_{2}{ }^{2}+R_{2}{ }^{2}$ and $Z_{4}=1+H_{4}{ }^{2}+R_{4}{ }^{2}$

$\varepsilon \quad$ emissivity of the heating element (-)

$\sigma \quad$ Stefan-Boltzmann constant, $5.67 \times 10^{-8}\left(\mathrm{~W} /\left(\mathrm{m}^{2} \mathrm{~K}^{4}\right)\right)$

$\omega_{2}, \omega_{4} \quad$ solid angles defined in Figure 1

FIRE SAFETY SCIENCE--PROCEEDINGS OF THE SEVENTH INTERNATIONAL SYMPOSIUM, pp. 815-827 


\section{INTRODUCTION}

The cone calorimeter (Babrauskas, 1982) was developed to test the response of materials exposed to a controlled level of radiant heating, as defined in a range of international (e.g. ISO 5660, 1993) and national (e.g. AS/NZS 3837, 1998) standards. Parameters such as ignitability, heat release rate, mass loss rate, heat of combustion, and smoke release of materials can be determined from experiments undertaken in the cone calorimeter. The heating element is rated as $5000 \mathrm{~W}$ at $240 \mathrm{~V}$, and consists of an element tightly wound into the shape of a truncated cone (frustum). The heater is designed to deliver irradiances on the surface of the specimen of up to $100 \mathrm{~kW} / \mathrm{m}^{2}$. It has been claimed that the unique design of the heating element is capable of producing radiant heat fluxes with uniformity of $\pm 2 \%$ within $50 \mathrm{~mm}$ by $50 \mathrm{~mm}$ area located $25 \mathrm{~mm}$ directly below the frustum.

The purpose of this work has been two fold. The primary objective is to examine the uniformity of the radiant heat flux striking the specimen's surface in the cone before ignition. However, our primary interest to study radiant heat fluxes in the cone calorimeter is related to intumescent materials. As these materials are exposed to fire, they swell and pyrolyse, creating a light porous structure that limits heat transfer to the substrate. In order to avoid contact of the advancing front of an intumescent material with the heating element, in the cone calorimeter, a retaining grid is placed on the material's surface prior to testing. Although useful for testing purposes, the grid limits the interface velocity in comparison to that expected in the field; for example, when an intumescent material coating an LPG bullet is exposed to a fire. By placing an intumescent material below the frustum at distances exceeding the standard $25 \mathrm{~mm}$, with no retaining grid, our aim is to investigate the incident flux history of the material's front, and to learn about its uniformity in the radial direction. With our focus on intumescent materials, this work differs from the measurements done by other researchers (Babrauskas and Parker, 1987; Scudamore et al., 1991; Lukas, 1995), who were concerned with the radiant heat flux inside the sample tray as the material's surface recedes in the fire process.

Consequently, the structure of the paper reflects these two objectives. The next section describes the geometry of the system and introduces the formula for the view factor. This is followed by a description of the experimental apparatus, and discussion of the results. Major findings of this work are summarised in Conclusions. In the paper, special attention has been given to estimate or calculate uncertainties in the experimental data.

\section{THEORETICAL}

Figure 1 illustrates the geometry of the interchange between the internal surface of the cone heater and an elemental area $d A_{1}$ located on the specimen's surface. From the position $d A_{1}$, the solid angle subtended when viewing area 3 corresponds to the difference in the solid angles $\omega_{2}$ and $\omega_{4}$ subtended when viewing areas 2 and 4 from $d A_{1}$, respectively. Thus the configuration factor $F_{d 1-3}$

$$
F_{d 1-3}=F_{d 1-2}-F_{d 1-4} .
$$


Physically, the configuration or view factor $F_{d 1-3}$ represents the fraction of the radiative energy leaving the elemental surface $d A_{1}$ of the specimen (in all directions) that reaches area 3.

The configuration factor for the interchange between the elemental area $d A_{1}$ and area 2 can be represented by the following expression (Siegel and Howell, 1992; see also Wang et al., 1986 for more complex geometries involving frusta)

$$
F_{d 1-2}=\frac{1}{2}\left(1-\frac{1+H_{2}^{2}-R_{2}^{2}}{\sqrt{Z_{2}^{2}-4 R_{2}^{2}}}\right),
$$

where $H_{2}=z / a, R_{2}=r_{2} / a$ and $Z_{2}=1+H_{2}{ }^{2}+R_{2}{ }^{2}$. A singularity exists in Equation 2 at the centreline, which necessitates expressing Equation 2 in a simplified form. Thus, for $a=0$

$$
F_{d 1-2}=\frac{r_{2}^{2}}{z^{2}+r_{2}^{2}} \text {. }
$$

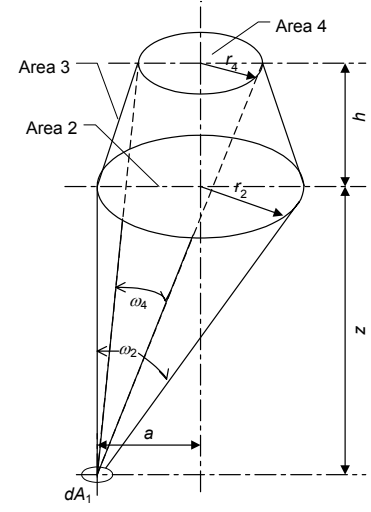

Figure 1. Schematic diagram of the internal surface of the frustum radiating to an elemental surface $d A_{1}$. Note that the heating spiral wound according to AS/NZS 3837 (1998) includes a short cylindrical section at the base of the frustum. The view factor (Equation 5) depends on $r_{2}, r_{4}$ and $h$, but not on the detailed shape of the heating element. Thus Equation 5 applies equally well to a heating element with and without the short cylindrical section.

If the analogues expressions are written for $F_{d 1-4}$, in conjunction with Equations 1-3 one obtains

$$
\begin{gathered}
F_{d 1-3}=\frac{1}{2}\left[\left(1-\frac{1+H_{2}{ }^{2}-R_{2}^{2}}{\sqrt{Z_{2}^{2}-4 R_{2}^{2}}}\right)-\left(1-\frac{1+H_{4}^{2}-R_{4}^{2}}{\sqrt{Z_{4}^{2}-4 R_{4}^{2}}}\right)\right], \text { and for } a=0 \\
F_{d 1-3}=\frac{r_{2}^{2}}{z^{2}+r_{2}^{2}}-\frac{r_{4}^{2}}{(z+h)^{2}+r_{4}^{2}}
\end{gathered}
$$

where $H_{4}=(h+z) / a, R_{4}=r_{4} / a$ and $Z_{4}=1+H_{4}^{2}+R_{4}^{2}$. 
Equations 4 and 5 have been plotted in Figure 2 using dimensions of the standard cone ( $h$ $=80 \mathrm{~mm}, r_{2}=80 \mathrm{~mm}$ and $r_{4}=40 \mathrm{~mm}$ ). The range of the abscissa covers the entire specimen's surface including the corners. Except very close to the frustum's base, $F_{d 1-3}$ decreases monotonically away from the centreline. Around the central portion of the specimen, $F_{d 1-3}$ does not vary substantially for distances close to (below $30 \mathrm{~mm}$ ) and far from (above $120 \mathrm{~mm}$ ) the base. However, between these two limits, the view factor rapidly decreases with distance below the frustum.

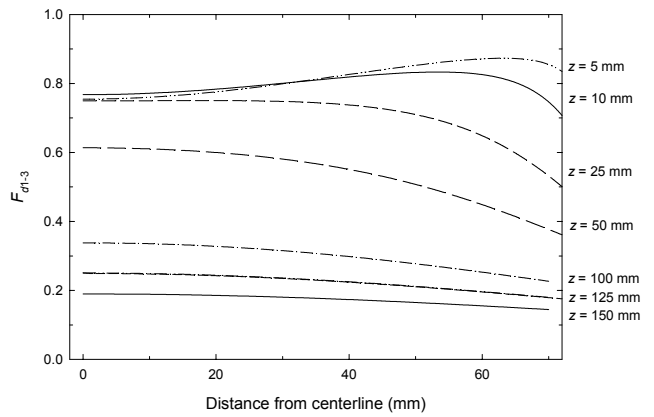

Figure 2. Variation in the cone configuration factor as a function of radial and axial coordinates.

Finally, the irradiance ( $q$ ") striking the specimen's surface at location $d A_{1}$ can be linked to the average temperature of the cone surface $(T)$ by

$$
q^{\prime \prime}=\frac{A_{3} F_{3-d 1}}{d A_{1}} \varepsilon \sigma T^{4}=F_{d 1-3} \varepsilon \sigma T^{4}
$$

where $\varepsilon$ and $\sigma$ have their usual meaning of surface emissivity and the Stefan-Boltzmann constant, respectively. The right equality in Equation 6 follows directly from the reciprocity relation of view factors $\left(A_{3} F_{3-d 1}=d A_{1} F_{d 1-3}\right)$.

\section{EXPERIMENTAL}

The experimental apparatus consisted of a cone calorimeter (FTT), Schmidt-Boelter gauge (Medtherm GTW-10-32-485A), an adjustable stand, and a heat-gauge positioning grid, machined from mild steel. The Schmidt-Boelter probe was $12.5 \mathrm{~mm}$ in diameter, circular in shape, coated with matt black finish, meeting the requirement of AS/NZS 3837 (1998). The centreline of the positioning grid was carefully aligned with the cone's axis. The positioning grid was bolted to an adjustable stand as sketched in Figure 3 . The $z$ coordinate was measured relative to the base of the frustum, whilst the $x$ and $y$ coordinates were measured relative to the cone centreline.

The positioning system consisted of 36 holes allowing the heat flux probe to be tightly fitted for measurements in each hole. The holes were drilled on a rectangular grid as illustrated in Figure 4; for example centre points of holes C4, C5 and C6 were located at positions $(7.14 \mathrm{~mm}, 7.14 \mathrm{~mm}),(21.42 \mathrm{~mm}, 7.14 \mathrm{~mm})$ and $(35.70 \mathrm{~mm}, 7.14 \mathrm{~mm})$, respectively. The grid was adjusted vertically with a tolerance of about $0.5 \mathrm{~mm}$. 


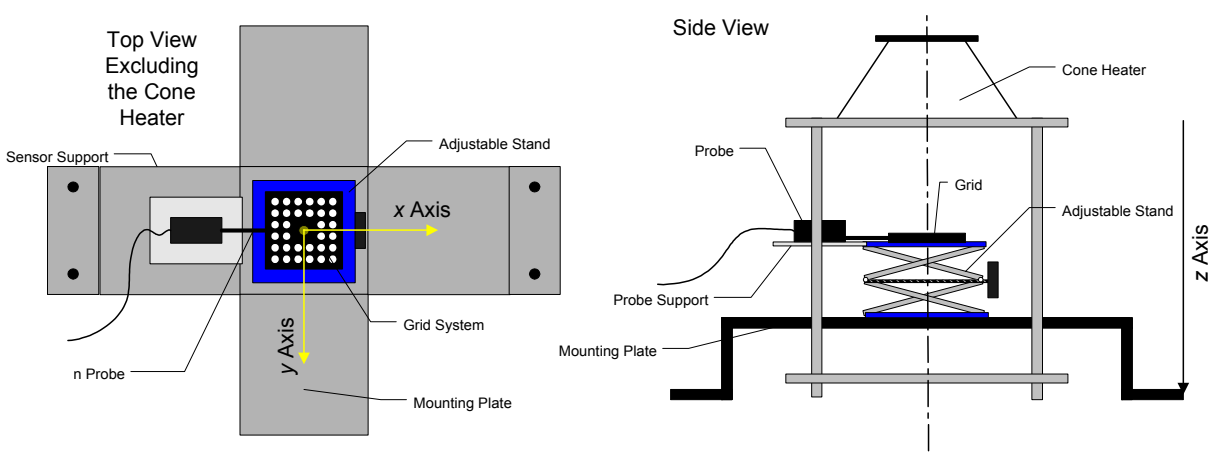

Figure 3. Sketch of the experimental apparatus, showing the heat flux sensor inserted into the middle hole in the positioning grid (left graph). The drawing on the right illustrates the location of the grid below the calorimeter's cone.

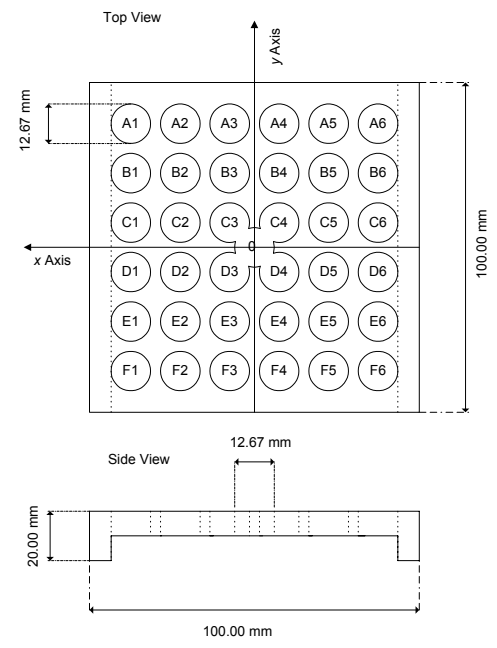

Figure 4. Detailed diagram of the positioning grid for the heat flux probe.

The relative error in $F_{d 1-3}$ corresponding to the uncertainty of $0.5 \mathrm{~mm}$ can be estimated from

$$
\frac{\Delta F_{d 1-3}}{F_{d 1-3}} 100 \%=\frac{\partial F_{d 1-3}}{\partial z} \frac{\Delta z}{F_{d 1-3}} 100 \%,
$$

where $\Delta z=0.5 \mathrm{~mm}$, with the partial differentiation on the right hand side of Equation 7 being carried out using Equation 5 along the centreline and Equation 4 off the centreline. Equation 7, plotted in Figure 5, demonstrates that the maximum relative error in the view factor, as a consequence of the uncertainty in the vertical adjustment of the probe, does not exceed 1\%. The error due to averaging effect of the sensing element of the SchmidtBoelter gauge is of the same magnitude as the error engendered by the uncertainty in the vertical adjustment of the probe. This conclusion comes from consideration of the 
average and local configuration factors for the Schmidt-Boelter sensors for small ratios of sensor to radiator areas (Figure 4 in Murthy et al., 1998).

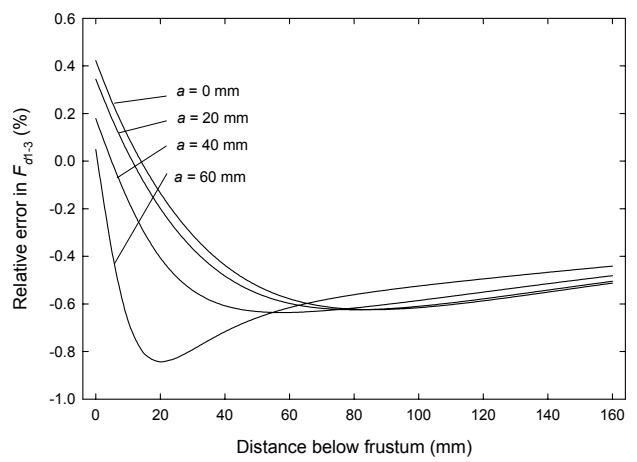

Figure 5. Plot of the relative error in the configuration factor due to the 0.5 $\mathrm{mm}$ tolerance in the vertical positioning of the probe.

Measurements at locations exceeding $35.70 \mathrm{~mm}$ along the $x$ and $y$ axes were required to produce isolines of the radiant heat flux and profiles of the heat flux as a function of the distance from the centreline. In these situations, the entire set-up, including the adjustable stand, was moved off the centreline to permit the collection of data. The heat flux was measured over seven horizontal planes that were located at 25, 35, 50, 75, 100, 125, and $150 \mathrm{~mm}$, relative to the base of the cone.

The emissivity of the heating element was estimated in the following manner: The temperature of the controller setting of the cone calorimeter was adjusted to a desired value and radiant heat flux was allowed to attain a steady state. A K-type thermocouple was inserted between two adjacent winding segments of the coil. The thermocouple was bent, with the thermocouple shield just after the bend made to touch cone-heating spiral for a distance of about $2 \mathrm{~cm}$. This was done to limit heat losses from the thermocouple junction. A Minolta pyrometer was aimed at the thermocouple location. The pyrometer emissivity was adjusted until the temperature displayed on the pyrometer was equivalent to that shown by the thermocouple. The data listed in Table 1 indicate the average emissivity of the heating spiral as $0.99 \pm 0.01$, where the uncertainty represents one standard deviation of the measurements. Note the difference between the cone controller temperature and the temperature measured by our thermocouple and the pyrometer. The temperature displayed by the cone controller is the average temperature obtained from three thermocouples permanently attached, from the back, to the cone heater.

All measurements presented in the next section of this manuscript have been collected for the temperature controller set at $650^{\circ} \mathrm{C}(923 \mathrm{~K})$. However, it is the average surface temperature of the coil, rather than the controller temperature, that must be substituted into Equation 6. In the first attempt to estimate the surface temperature of the coil, we have divided the inside surface of the frustum into 12 equal-area segments, and measured their average temperatures. The results obtained $(918,918,893,900,966,930$, 903, 933, 945, 929, 945 and $933 \mathrm{~K}$ ) demonstrated substantial scatter in the surface temperature; $T=926 \pm 20 \mathrm{~K}$, corresponding to $8 \%$ uncertainty in the incident heat flux along the centreline. To assess the predictive power of the model, a smaller uncertainty is desirable. In the second approach, we calculated $q$ " at $25 \mathrm{~mm}$ below the frustum along the centreline from four measurements conducted at locations C3, C4, D3 and D4 (see Figure 4) to obtain $32.7 \pm 0.2 \mathrm{~kW} / \mathrm{m}^{2}$. By applying Equation $6\left(F_{d 1-3}=0.746\right.$ at this location; see 
Figure 2), we have calculated $T=940 \pm 1 \mathrm{~K}$, which will be used to compare measurements with the theory in the remaining part of the paper.

Table 1. Emissivity of the cone-heating element as a function of the cone temperature setting and local temperature reading. Although the temperature values of the cone controller are listed in ascending order, they were randomised in the experiments.

\author{
Cone controller \\ (average) temp, K
}

Thermocou
pyrometer (local)
745
881
860
915
923
951
992

Emissivity, -

0.980

1.000

0.970

0.985

1.000

0.985

0.990

Average: $\quad 0.99 \pm 0.01$

\section{DISCUSSION}

Figure 6 presents the isolines of the radiant heat fluxes at various heights below the frustum's base. These data correspond to the specimen's surface at the top of the sample tray. The results have practical application to situations where a specimen would have been mounted at heights different from the standard $(25 \mathrm{~mm})$, as it could be the case for experiments with intumescent materials, with no retaining grid. The experimental isolines were generated from 50-point measurements at each height; 36 points were taken when the positioning grid was located exactly along the cone's centreline (as shown in Figure 3 ), with the additional 6 measurements taken in each direction by moving the positioning grid (thus 24 readings). Although, good agreement has been obtained between the theoretical and experimental isolines, the shapes of the experimental isolines are not as regular as those obtained from Equation 4. There are three reasons for this behaviour.

Calculation of the radiant fluxes using the view-factor algebra assumes a uniform distribution of radiosity on the inside surface of the frustum. In practice, this assumption is satisfied only approximately. As shown in the previous section, the surface temperature of the heating spiral may deviate substantially from its average value. This translates to a non-uniform distribution of radiosity and hence also irradiance. The second reason is due to the radiant heat flux from the hot open shutter, which has not been taken into account in the theoretical analysis. The heat flux originating from the shutter can increase the irradiance, especially toward the specimen's edges where the effect can be as high as $6 \%$ of the total heat flux, as is illustrated in Figure 7 for $z=0.1$ and $0.15 \mathrm{~m}$. Also, some radiation, arguably small, may reach the sensor by reflection from various surfaces of the cone calorimeter. Finally, convection effects due to the heating of the positioning grid may influence the radiant heat flux measurements. These effects have not been studied in this work, as it was thought that they are minimised by buoyancy. However, the convection effects around Schmidt-Boelter sensors have been found to be significant in 
other geometries (Murthy et al., 1999). At present, a new generation of heat flux sensors are being developed which are able to measure radiant and convective contributions to the total heat flux (e.g. Martins et al., 2000). Unfortunately, these sensors have not yet been applied in cone-calorimetric studies.
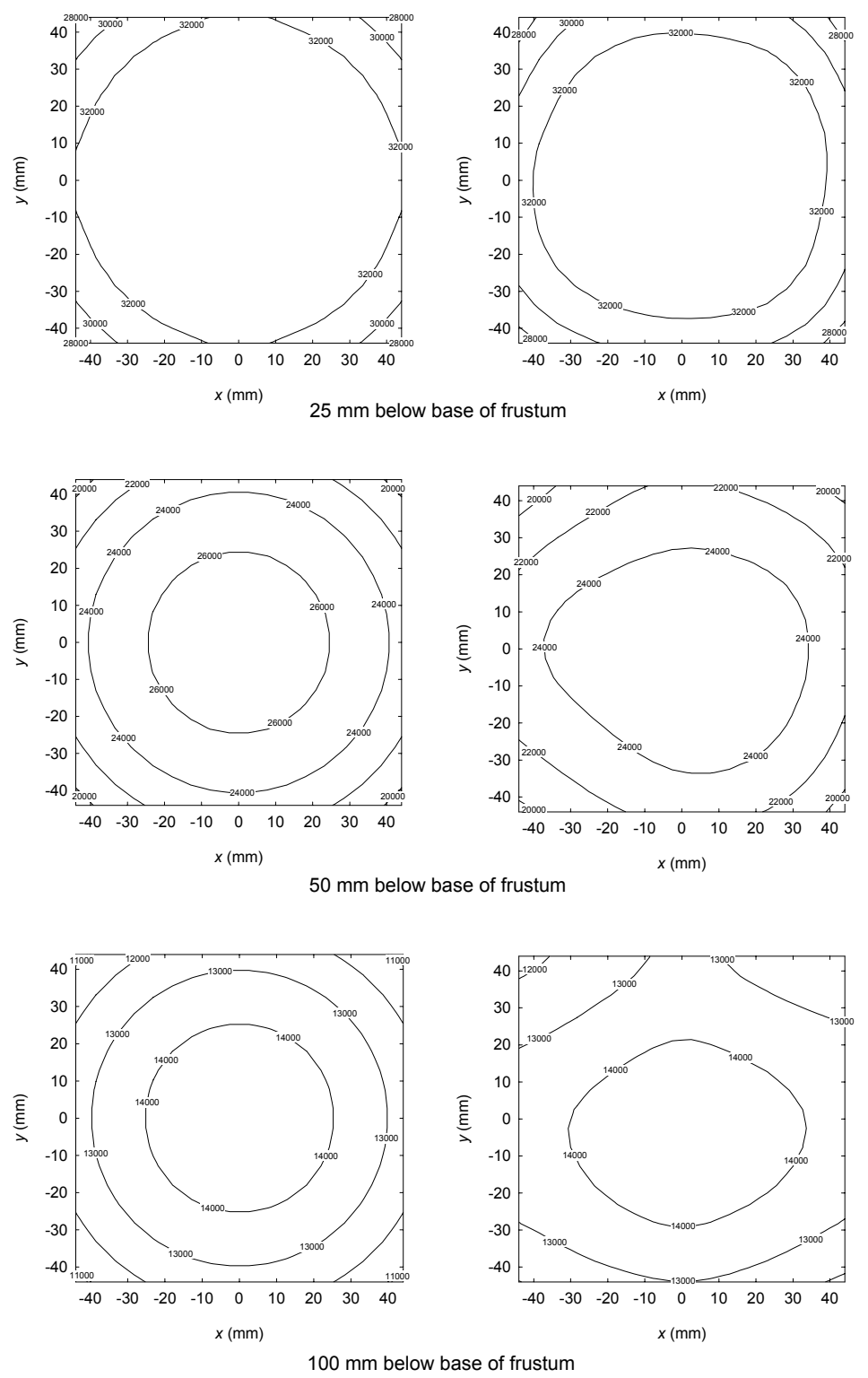

Figure 6. Isolines of the radiant heat fluxes, the theoretical predictions are illustrated on the left and the experimental data are shown on the right; $T=940 \mathrm{~K}$. 
The data plotted in Figure 6 have been summarised in Figure 7, as a function of the radial coordinate. As one moves away from the centreline, the scatter increases owing to the decreasing axial symmetry of the isolines, though there is overall good agreement between the results of view-factor calculations and measurements. Application of Equations 4 and 5 allow us to obtain an estimate of the difference in the local values of the radiant heat flux. For example, at $z=25 \mathrm{~mm}$ below the frustum, the theoretical difference between the centre and the edge (taken as $44 \mathrm{~mm}$ from the centre) is $2.6 \%$ and between the centre and the corners (taken as $62 \mathrm{~mm}$ from the centre) is $15.9 \%$. However, within the central $50 \times$ $50 \mathrm{~mm}$ area of the specimen the variation in the radiant heat $(0.7 \%)$ is well within that specified by the ISO 5660 (1993) standard of $\pm 2 \%$.

Figure 8 demonstrates an interesting behaviour of the radiant heat flux (along the centreline) which is observed to decrease with smaller distances below the frustum. This can be explained with reference to solid angles drawn in Figure 1: As $z$ decreases $\omega_{4}$ becomes more significant than $\omega_{2}$, leading to the reduction in $F_{d 1-3}$. The experimental points shown in Figure 8 have been collected slightly off the centreline at positions C3, C4, D3 and D4 (Figure 4). Finally recall that, the average temperature of the spiral surface was determined by applying Equation 6 at $z=25 \mathrm{~mm}$. For this reason, there is no difference between the calculated and (average) experimental results at this point (see inset in Figure 8).

Figure 9 explores the effect of a deviation in frustum dimension from the standard geometry on the configuration factor. Small changes in frustum height that occur as a consequence of cone sagging, when operated at high fluxes $\left(90-100 \mathrm{~kW} / \mathrm{m}^{2}\right)$ for prolong periods of time, lead to errors of less than $3 \%$ in $F_{d 1-3}$, its magnitude decreasing with increasing distance below the base of the frustum (Figure 9a). Similarly, small changes in the radius of the base of the frustum results in small errors in the configuration factor, but only for the central area of the specimen. Close to the specimen's edges and corners, the magnitude of the errors increase significantly, as illustrated in Figure 9b. The data presented in Figure 9 are quite important when considering deformations that can occur in the cone-heating spiral.

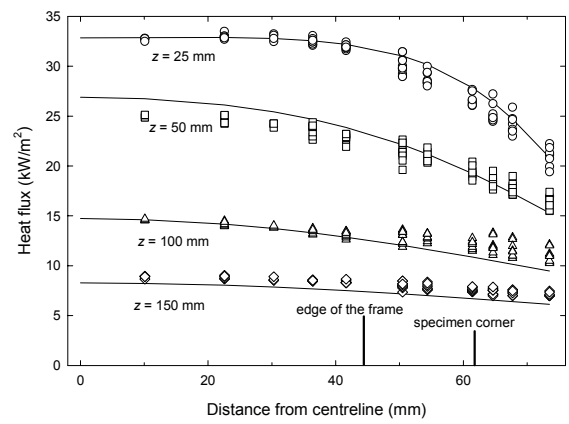

Figure 7. Measured and calculated radial variation in the radiant heat flux for average surface temperature of the heating spiral of $940 \mathrm{~K}$.

In all experiments reported in this work, no specimen tray has been used. Thus the results presented here correspond to the distribution of radiant heat flux on the specimen's surface prior to ignition, for experiments conducted at the standard $(z=25 \mathrm{~mm})$ and nonstandard $(\mathrm{z} \neq 25 \mathrm{~mm})$ distances below the cone.

The present results can also be used for intumescent materials which swell and grow out of the sample tray. However, for many materials studied in the cone calorimeter, the specimen's surface recedes with time after ignition. For these materials, 
one would like to know the distribution of irradiance at various heights within the experimental tray. This problem has been studied by other researchers with results reported in the literature but only for radiant heat fluxes along the centreline.

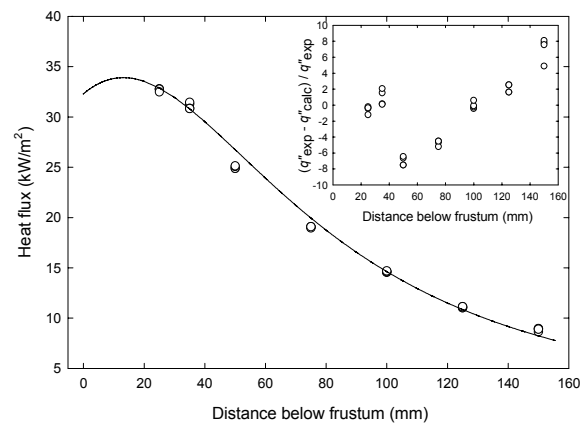

Figure 8. Comparison between the calculated and measured radiant heat fluxes along the centreline; $T=940 \mathrm{~K}$.

In particular, Lukas (1995) has measured irradiances within the sample tray with and without edge frame. Her data indicate that the sample tray acts as a trap to the incoming radiation, resulting only in a very small variation in the radiant heat flux with height within the tray. This is illustrated in Figure 10. For the purpose of comparison, the ordinate axis corresponds to $q^{\prime \prime}(z) / q "(z=25 \mathrm{~mm})=F_{d 1-3} /(z=25 \mathrm{~mm})$. In this plot, Lukas' data are drawn together indicating that sample tray affects the radiant fluxes by the same mechanism irrespective of the heat-flux level. The impact of the sample tray on maintaining the nominal irradiance levels along the centreline is very significant.

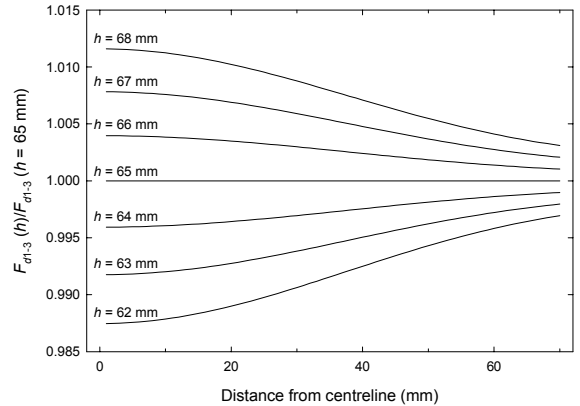

Figure 9a

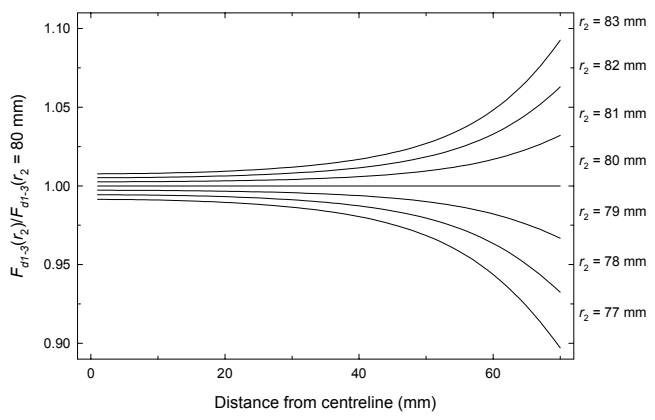

Figure 9b

Figure 9. Comparison of the view factors for cone geometries differing slightly from the standard dimensions; (a) the effect of cone height and (b) the effect of frustum's base radius. 


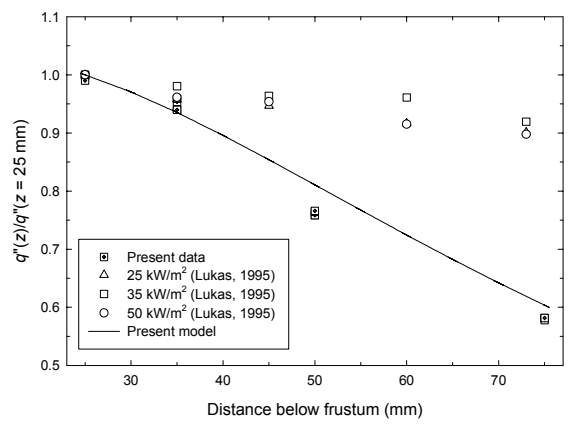

Figure 10. The effect of sample tray on irradiances along the centreline. The present data collected with no sample tray are compared with Lukas' results obtained within a sample tray surrounded by an edge frame.

\section{CONCLUSIONS}

This study has shown that the radiant heat flux under the cone heater can be accurately described for engineering applications by the following expression

$$
\begin{gathered}
q^{\prime \prime}=\frac{1}{2}\left[\left(1-\frac{1+H_{2}{ }^{2}-R_{2}^{2}}{\sqrt{Z_{2}^{2}-4 R_{2}^{2}}}\right)-\left(1-\frac{1+H_{4}^{2}-R_{4}^{2}}{\sqrt{Z_{4}^{2}-4 R_{4}^{2}}}\right)\right] \varepsilon \sigma T^{4} \text { or as } \\
q^{\prime \prime}=\left[\frac{r_{2}^{2}}{z^{2}+r_{2}^{2}}-\frac{r_{4}^{2}}{(z+h)^{2}+r_{4}^{2}}\right] \varepsilon \sigma T^{4}
\end{gathered}
$$

along the cone's centreline. In these equations, $\varepsilon$ is the emissivity of the surface of the heating coil and $T$ is its average temperature. Other symbols occurring within the square brackets are geometric parameters defined in Nomenclature and in Figure 1. These equations do not apply within the sample tray.

In this study, the surface emissivity of the heating element was estimated as 0.99 \pm 0.01 . The surface temperature was always found to be above that displayed by the cone's temperature indicator. The average surface temperature of the heating spiral can be readily calculated from a single calibration measurement along the centreline using the latter of the above equations. This temperature can then be substituted into the expression for $q$ " to obtain accurate predictions of the incident heat flux at any location under the frustum.

Small differences between the measured and calculated irradiances were observed and explained by non-uniform distribution of radiosity on the surface of the heating coil, radiant fluxes from hot components of the cone assembly (e.g. the shutter) and reflected radiation, as well as convective heat transfer effects on the operation of the Schmidt-Boelter's sensor. Relative uncertainties in the radiant flux due to the averaging effect of the probe, tolerance in the vertical positioning of the probe and minor modifications in the heating-element geometry are small and do not exceed a few percent.

Variation in the local configuration factor leads to differences between the radiant heat flux received by the central area of the specimen and its edges, and between the central area and the corners. As predicted by the model and confirmed by experiments, at $25 \mathrm{~mm}$ below the frustum, these differences are in the order of 2.6 and $15.9 \%$, respectively. However, within the central $50 \times 50 \mathrm{~mm}$ area of the specimen the 
variation in the radiant heat $(0.7 \%)$ is well within that specified by the ISO $5660(1993)$ standard of $\pm 2 \%$.

\section{ACKNOWLEDGEMENTS}

This study has been funded jointly by the Australian Research Council and STR Applications Pty. Ltd. through a Linkage Grant to the University of Newcastle. The authors are grateful to Mr. Chris Allen, Dr. Atul Bhargava and Prof. Michael Delichatsios for their contributions to the present investigation.

\section{REFERENCES}

AS/NZS 3837 (1998) Method of Test for Heat and Smoke Release Rates for Materials and Products Using an Oxygen Consumption Calorimeter, Council of Standards of Australia.

Babrauskas V. (1982) Development of the Cone Calorimeter: A Bench Scale Heat Release Rate Apparatus Based on Oxygen Consumption (NBSIR 82-2611), NIST Gaithersburg, MD.

Babrauskas V. and W.J. Parker (1987) "Ignitability measurements with the cone calorimeter", Fire \& Materials 11, 31-43.

ISO 5660 (1993) Fire Tests; Reaction to Fire; Part 1: Rate of Heat Release from Building Products (Cone Calorimeter Method).

Lukas C. (1995) "Communication: Measurement of heat flux in the cone calorimeter", Fire \& Materials 19, 97-8.

Martins N., M. G. Carvalho, N. Afgan and A.I. Leontiev (2000) "A radiation and convection fluxmeter for high temperature applications" Exp. Therm. \& Fluid Sci. 22, 165-73.

Murthy A. V., B. K. Tsai and R. D. Saunders (1998) “Aperture proximity effects in high heat flux sensors calibration" J. Res. NIST 103, 621-4.

Murthy A. V., B. K. Tsai and R. D. Saunders (1999) "Comparative calibration of heat flux sensors in two blackbody facilities" J. Res. NIST 104, 487-94.

Scudamore M.J., and P.J. Briggs and F.H. Prager (1991) "Cone calorimetry: A review of test carried out on plastics for the Association of Plastics Manufacturers in Europe" Fire \& Materials 15, 65-84.

Siegel R. and J.R. Howell (1992) Thermal Radiation Heat Transfer, Hemisphere Publishing Corporation.

Wang J. C. Y., S. Lin, P.-M. Lee, W.-L. Dai and Y.-S. Lou (1986) "Radiant interchange configuration factors inside segments of frustum enclosures of right circular cones" Int. Comm. Heat Mass Transfer 13, 423-32. 